omitted, as it does not agroe; and the solution of the chloride of lime to be continued.

9. Berotal and perineal ulcers healed, whilst that on the pubes is enlarged. Hair to be removed, and the sore treated with a solution of the sulphate of copper (gr. $x$ ad $3 j$ ); gr. viij of the iodide of potassium ter die, and $\mathrm{gr}$. $v$ of the blue pill every night.

Dec. 15th. Much better in every respect. Ulcer on the pubes rapidly cicatrising.

Dec. 26th. Going on well. Ulcer on the pubes nearly healed.

Dec. 29th. Discharged cured.

This appears to be a case of primary syphilide, and of a phagedenic character. I believe it was right, under the circumstances, to give mercury in the first place; but, as the morbid action of the sores did not yield to this remedy, it became necessary to try others till the rapid progress of the disease was checked; and this was effectually done by large doses of the iodide of potassium.

It may be asked, What gave rise to this syphilide? Mr. Pearson is of opinion that syphilides are not communicated by lues, as they often occur where the female is not affected with it; but this opinion seems to be at variance wish what he states elsewhere-that persons who lead correct lives are never affected with syphilides. The true explanation is probably this-that syphiloid disease is either communicated by the same poison, or that it is caused by contact with true syphilis, and converted into a complicated or syphiloid disease by the peculiar constitution or condition of the recipient.

\section{CASE OF LITHOTOMY PRESENTING UNUSUAL DIFFICULTIES, AND ONE OF STAPHYLORAPHY.}

By AUGUSTIN PRICHARD. Esq., Surgeon to the Bristol Royal Infirmary.

[Read at the Meeting of the Bath and Bristol Branch, November 15th, 1855]

TuE following surgical cases appear to be worth bringing before the Boeiety, on account of some accompanying unusual circumstances.

CAse I. Calculus Vesicce. E. Hembury, aged 34, a hairdresser by occupation, short in stature, with the bones of his legs curved by old rickets, and presenting otherwise an unhealthy strumous aspect, was admitted as my patient at the Bristol Royal Infirmary early in September. He has suffered severely from stone symptoms for five years; but his mother says that he has always been subject to some complaint about his urinary organs.

On the 7 th of September I introduced the lithotrite (of course, after having detected the stone by a sound,) and made an attempt to lay hold of the stone; but I could not catch it; and, although I could expand the blades freely, it did not fall down upon the instrument, as has happened in some cases in which I have crushed the stone, but appeared to remain in front of the bladder. The points of the blades of the lithotrite were covered with minute portions of the calculus, against which they had rubbed.

As this attempt failed, after consultation, I performed lithotomy. The patient was placed under the influence of chloroform, with an hour's secretion of urine in his bladder. I then introduced the staff, and tied him up. After the first incisions were made, I cut along the groove in the staff in the usual way; but the instrument had slipped out of the bladder, and consequently the knife did not enter its cavity at once. Fortunately, I managed to reintroduce it, and complete the section in the prostate without any loss of time. Upon the introduction of the finger, I could feel the stone; but it did not fall down, and as it were follow the finger to the wound, as is generally the case, but it remained above, and I had to introduce my finger as far as I could, backwards and upwards, to the point where the stone presented itself, much in the position of a child's head high up in the pelvis. I had hold of the stone sereral times, and used immense force, but it did not move; and in fact, I could only grasp it by opening the forceps, and in fact, I could only grasp it by opening the forcible pressure above the pubis by which the stone was brought within reach of the pubis, by which the stone was brought within reach of the mastrumeced in cracking it with a screw forceps; and mately succeeded in piecemeal; but it was a tedious and laborious process. But ultimately it was all removed; and when the largest portion was still in situ, I found that with the fingers I could separate it from the mucous membrane of the anterior part of the bladder.

The patient, after the cavity had been washed out with two or three syringes full of hot water, was carried to bed in a very exhausted state, having lost a considerable quantity of blood, and having been nearly an hour under chloroform. A dose of brandy with opium was given him, and he soon went to sleep.

The next day, he complained of some hypogastric pain; but the urine had flowed freely through the wound, and he was as well as he could be after the fearful ordeal he went through the day before. He required stimulus from the first, but he very gradually and slowly mended. The wound gradually closed, but no urine came through the urethra until thirty-eight days after the operation. The chief drawback was the obstinate constipation from which he suffered, and which, in spite of a good diet, wine, castor oil, colocynth pills, infusion of roses and sulphate of magnesia in repeated doses, and gruel and pulp of colocynth injections, all of which he retained, lasted sixteen days, and ultimately yielded to an injection of half an ounce of fresh oxgall in a pint of gruel.

He went out at the end of last month, in a reak state, but able to sit up, and having quite recorered from the immediate effects of the operation. I have since heard that he remains weak; and a sample of his urine, which was brought to me, showed a considerable quantity of pus.

The stone was, I am convinced, adherent to the anterior surface of the bladder; and this, in addition to its unusual size, constituted the difficulty of the operation. The case, if occurring to a surgeon operating for stone for the first time, would have been enough to discourage even the most sanguine; and it illustrates, I think, one of the adrantages of chloroform; for 1 cannot imagine that, without it, any man could have borne the protracted manipulations of no gentle kind, to which the poor fellow was necessarily subjected. The stone weighed $2+$ ounces and 30 grains.

CAsE II. Staphyloraphy. The next case is that of a young man in whom I performed the operation of sewing up the soft palate. It was a congenital fissure, which had extended through from the lip to the end of the uvula; but the hare lip had been cured in his infancy. His speech is extremely defective and occasionally the fluids he drinks escape from the nose. The patient was by occupation a farmer, and was 24 years of age. I directed him to make a good breakfast, as he would have to starve for a day or two after the operation; and, on going to perform it, at $11 \frac{1}{2}$ A.M. On the 25th of September, I found that he had complied with my request, by taking, besides his breakfast, a lunch composed of the greater share of two pounds of beef-steak and the proportionate quantity of beer.

With a long forceps, I held the top of the left subdivision of the urula ; and then, with a narrow and straight bistoury, pierced it, and stripped off the free edge of the velum pendulum; and performed exactly the same on the other side. With the aid of the instrument which I show you, which lays hold of the thread and draws it through, I was able easily to pass the suture through, holding, for the first stitch, each end of the uvula; for the second stitch, the first was the most convenient means of steadying the parts; and, in introducing this, I was enabled to run the curved needle through both sides at the same time." I tied the anterior stitch with the aid of a piece of stick notched at the end: the second I tied far more conveniently with my fingers. He was of course coughing and hawking and spitting during these manæurres; but, fortunately, the re- 
Sax sction was not atrong enough to bring up the contents $\alpha$ his stomach. He went to bed, and slopt most of the an: : in fact, he had no subsequent trouble, except a little firting from codems of the uvula.

I took out the stitches on the $28 \mathrm{th}$, and found that the wound had entirely healed; and so it remained. Of course the bony aperture still remains to be relieved by artificial means.

\section{CASE OF SUICIDE BY CUT THROAT.}

\section{By JOHN S. BARTRUM, Surgeon to the Bath General Hospital.}

[Read at the Meeting of the Bath and Rristol Branch, November 15th, 1855.]

A smawe woman, aged 52 years, of habitually saving habits, and genuinely religious sentiments, in the spring of 1855 was attacked with chronic pleuropneumonia, accompanied with much pain in the side, and expectoration. Until this period she had always been characterised by shrewdness, firmness, and good sense; she now became the subject of many delusions; would continue unclothed during the intense cold; would unceasingly spit, to get rid, as she said, of the inflammation; slept very little, and was occupied with divers vagaries, for each of which she had some apparent reason. I first saw her when convalescent: as the state of her lungs gradually improved, so her mental ailments decreased, till, after some weeks, she wholly recovered her bodily and mental powers.

In the earlier part of her illness she had not been managed by her nurse with sufficient firmness, which, I have no doubt, materially aggravated her bodily ailments, and so added to her mental hallucinations.

For some months she continued quite well, and able to resume her duties as confidential lady's maid. In this interval she often spoke without reserve of her previous state of mind, saying that she could not control her shouting, laughing, singing, etc.; and that, though she had been threatened by her previous medical attendant with being sent to a lunatic asylum, she stated that she was quite sane but could not control herself; this deficiency of self-control, however, from my own experience of her, I much doubt, had she at that period been properly managed by her nurse.

After a prolonged sojourn at the sea side, she returned to her usual residence, performed more than her ordinary duties most efficiently, and went to bed with her mistress. The morning after her return, her fellow servant found her sitting on the floor of the scullery half dressed with her throat cut on the left side. It seemed that she had left her mistress's bed, come down three flights of stairs, procured one of the ordinary blunt kitchen knives, and inflicted an awful gash; kneeling before the dust-box, she coolly held her head over it, supporting herself on it with both her hands; she had put the knife on the floor, and then left herself to bleed: for some reason she had seated herself on the floor, where she was found. Probably she partially fainted and then turned round. Between the box and the place where she was seated, there were but few spots of blood, and these close to the box, the blood having probably run down her neck as she turned round. She was then helped up, and walked or staggered about four feet farther, still leaving no tracks of blood. At her express desire, she washed her hands and partially her face at a sink close at hand. On the outside of the dust-box were bloody thumb marks, and bloody finger marks on the inside, but not a drop was spilt on the floor. Her mental state, as far as we could judge by expression, was one of intense agony ; her appeals to heaven for mercy were continuous. Afler some hours she was able to be removed to the hospital, where she recovered sufficiently to express herself by writing. From her statements there, as well as from her previously expressed fears, it seemed that she dreaded leaving her situation and coming to porerty, both of which were groundless, she being very much trusted, and having comfortable independent means. This mentel condition struck me as very singular; to the last her coenscience appeared to be in full exercise, as she evidently looked for mercy where alone it is to be sought-where she had been accustomed to appeal; at the same time she de liberately wrote down that she had no money, that she must go to the poor house, though the previous day she had given her friend thirty pounds to take charge of, and much money was found in her possession. In this state she continued, till increasing dyspncea induced insensibility and death, having lived upwards of seventy hours.

On examination, the incision commenced to the right of the median line, she had severed wholly the trachea, dividing two of the upper rings; the esophagus was cut across, as was the jugular; there was a slight orifice in the carotid, scarcely more than a pin hole; the condition of the pneumogastric was not noted, and the intervertebral substance between the fourth and fifth cervical bones was very deeply divided.

This case appeared to me worthy of record from two reasons, one medico-legal, the other psychological.

When I first saw her, shortly after she was found, there was ouly a general oozing of not florid blood from the wound, every expiration forcing up the bloody froth; so soon after the injury as when she was at the dust-box, it struck me as remarkable, that with such a wound in such a situation, she could move a distance of five feet without leaving traces of blood, and there were none, as I at once looked for them carefully. Her mental condition was equally remarkable. For several days before her return she had been desponding and altered, full of fears about her mistress's displeasure-of fears about her poverty, etc. The meditated deed is effected; she becomes fully aware of its iniquity, yet the delusion continues unshaken to the end. Whether this be an ordinary state in those labouring under any mental delusion I do not know; but if the case elicit any observations, or call forth the experience of the members having larger fields of obserration, it will have fulfilled its purpose.

\section{THE WEATHER.}

By J. A. HiNgeston, Esq., Brighton.

Arter the very long drought, which has characterised nire wonths of the present year, the rain that has lately fallen, between September 27 th and November 7 th following, has amounted, in this locality, which is 200 feet above the level of the sea, to the depth of 7.68 inches. The amount that fell from the beginning of the year up to June 30 th, was only 6.28 inches, melted snow included. In July, 3.54 inches fell, and in August, 0.71 of an inch. When the south-westerly gales prevail in the autumn, a great deal more rain falls here than farther inland, owing to the clouds, surcharged with moisture from the Atlantic, depositing their load of vapour in floods, the moment they break along this line of coast.

The temperature has hitherto been high; and the gales from the S.W. account for the warmth, together with the south facing, of this locality. The average range of the thermometer may be stated at from $50^{\circ}$ to $55^{\circ}$ of Fahrenheit. Sometimes it has risen above this altitude, but it has seldom fallen below it. On the morning of the 4th Norember, it stood at $38^{\circ}$, and there was some sleet, the wind being northerly.

On the 26th October, there was a hurricane from the W.S.E., which lasted twenty-four hours. It subsided all of a sudden, just at high-tide, about 10 A.M., on the 27th. The eclipse of the moon on the 25th was hidden behind the driving scud. The flood-tides, driven to shore by the wind, were very high. The barometer rose before the storm burst, and stood at 30 inches; it then fell rapidly down to 29.048. The thermometer raried between $63^{\circ}$ and $50^{\circ}$ Fahrenheit. The electrometer was capricious. The magnet undisturbed. Aftex the storm, the thermometar ank 